Research

\title{
Evaluating the reliability, validity and minimally important difference of the Taiwanese version of the diabetes quality of life (DQOL) measurement
}

\author{
I-Chan Huang*1, Jung-Hua Liu ${ }^{1}$, Albert W Wu ${ }^{2,3}$, Ming-Yen $\mathrm{Wu}^{4}$, \\ Walter Leite ${ }^{5}$ and Chyng-Chuang Hwang ${ }^{4}$
}

\begin{abstract}
Address: ${ }^{1}$ Department of Epidemiology and Health Policy Research, College of Medicine, University of Florida, Gainesville, FL, USA, ${ }^{2}$ Department of Health Policy and Management, Bloomberg School of Public Health, Johns Hopkins University, Baltimore, MD, USA, ${ }^{3}$ Department of Medicine, School of Medicine, Johns Hopkins University, Baltimore, MD, USA, ${ }^{4}$ Tainan Hospital, Department of Health, Tainan, Taiwan and ${ }^{5}$ Department of Educational Psychology, College of Education, University of Florida, Gainesville, FL, USA

Email: I-Chan Huang* - ichuang@ufl.edu; Jung-Hua Liu - jhliu1115@hotmail.com; Albert WWu - awu@jhsph.edu; Ming-

YenWu - mingyenwu@ymail.com; Walter Leite - Walter.Leite@coe.ufl.edu; Chyng-Chuang Hwang - chyngc.hwang@msa.hinet.netail.com

* Corresponding author
\end{abstract}

Published: 28 October 2008

Health and Quality of Life Outcomes 2008, 6:87 doi:10.1 186/1477-7525-6-87
Received: 20 March 2008

Accepted: 28 October 2008

This article is available from: http://www.hqlo.com/content/6/1/87

(C) 2008 Huang et al; licensee BioMed Central Ltd.

This is an Open Access article distributed under the terms of the Creative Commons Attribution License (http://creativecommons.org/licenses/by/2.0), which permits unrestricted use, distribution, and reproduction in any medium, provided the original work is properly cited.

\begin{abstract}
Background: Few diabetes HRQOL instruments are available in Chinese language. We tested psychometric properties of a Diabetes Quality of Life (DQOL) in Chinese language for diabetes patients in Taiwan and estimated its minimally important differences (MIDs).

Methods: Data were collected from 337 patients treated in diabetes clinics of a Taiwan teaching hospital. Pearson's correlations among domain scores of the DQOL (satisfaction, impact, and worry), the D-39S (a diabetes-specific instrument, including domains of diabetes control, energy and mobility, social burden and anxiety and worry, and sexual functioning) and the RAND-12 (a generic instrument, including physical health composite (PHC) and mental health composite $(\mathrm{MHC})$ ) were estimated to determine convergent/discriminant validity. Known-groups validity was examined using 2-hour postprandial plasma glucose (2 h PPG), hemoglobin Alc (HbAlc)) and presence of complications (retinopathy, neuropathy, and diabetic foot complications rather than the known groups of cardiovascular and cerebrovascular complications). We used a combined anchor- and distribution-based approach to establish MIDs.
\end{abstract}

Results: The DQOL scores were more strongly correlated with the physical domains of the D$39 \mathrm{~S}$ (diabetes control and energy and mobility) and RAND-12 PHC than psychological domains of the D-39S (social burden, anxiety and worry, and sexual functioning) and RAND-12 MHC. The DQOL showed satisfactory discriminative ability for the known groups of $2 \mathrm{~h}$ PPG and HbAlc (effect size $(E S) \geq 0.2$ ) and retinopathy, neuropathy, and diabetic foot complications ( $E S \geq 0.3$ ), but less satisfactory for the known groups of cardiovascular and cerebrovascular complications. MIDs for the DQOL domains were 3-5 points for satisfaction, 4-5 points for impact, 6-8 points for worry, and 3-4 points for overall HRQOL.

Conclusion: We validated a DQOL in Chinese language for diabetes patients in Taiwan and provided MIDs to facilitate the measure of diabetes HRQOL. 


\section{Background}

Diabetes mellitus (DM) is associated with long-term damage of multiple organ systems and increased age-adjusted mortality rates. Conventional assessment for diabetic patients relies on clinical measures, e.g., glycemic control and diabetes complications. However, the use of clinical measures alone for diabetes management is limited because clinical measures can not fully capture patient's health outcomes, especially psychological impact [1]. Health-related quality of life (HRQOL) measures, which emphasize daily functioning and well-being, are useful adjuncts to clinical indicators for assessing diabetic health outcomes.

Several instruments are available for assessing diabetes HRQOL, including generic and diabetes-specific instruments. Generic instruments measure HRQOL domains which are universally important across diseases, while diabetes-specific instruments measure specific impacts of diabetes on functioning and well-being. Specific instruments may be more sensitive to patients' score changes over time $[2,3]$.

There is a great need to develop and validate diabetes HRQOL instruments for Chinese populations, which comprise the largest group of people with diabetes [4]. Although more than a dozen of diabetes HRQOL instruments have been developed $[5,6]$, only three instruments are available in Chinese language (including a translated Diabetes-39 (D-39) [7] and a translated Diabetes Impact Measurement Scales (DIMS) [8] for Chinese people in Taiwan, and a translated Diabetes Quality of Life (DQOL) [9] for Chinese people in Canada). Each instrument, however, may measure somewhat different concepts of HRQOL. For example, the D-39 measures the concepts of physical functioning and psychosocial well-being associated with diabetes including the domains of energy and mobility, diabetes control, anxiety and worry, social burden, and sexual functioning [10], whereas DQOL measures the burden associated with diabetes treatment and glycemic control including the domains of satisfaction with treatment, impact of treatment, and worry about future effect of diabetes [11]. Therefore, it is important to validate and compare different instruments within the same population and to test whether one instrument may be used combined with another to better capture comprehensive diabetes HRQOL.

In testing the usefulness of diabetes HRQOL instruments, the selection of psychometric methods and clinical variables can influence the success of instrument validation [7]. Hemoglobin A1c (HbA1c) - a measure reflecting a longer-term glycemic control - is commonly used as an external variable to validate instruments, but the association between HbA1c and HRQOL is weak [12]. Validation might be improved by further including other laboratory indicators (e.g., fasting plasma glucose (FPG) and postprandial plasma glucose (PPG)) to better account for the impact of fluctuations and acute increase of glycemia (hyperglycemic spikes) on health $[13,14]$. Additionally, hyperglycemic symptoms and diabetic complications are major determinants of HRQOL $[15,16]$. The use of laboratory indicators (FPG, PPG and HbA1c) together with diabetes complications would be helpful for validating diabetes HRQOL instruments.

An issue limiting the use of diabetes HRQOL measures is that little guidance is available to interpret HRQOL scores, especially clinical meaning in score difference among treatment groups or score change within individuals over times $[5,17]$. Conventionally, the interpretation of score changes/differences relies on tests of statistical significance. Yet, statistical significance is not equivalent to clinical significance because the former does not directly link to clinical sensibility and is partially determined by sample size [3]. Clinicians are interested in interpreting score differences, especially minimally important difference (MID) which can serve as the lowest benchmark to determine clinical meaning of HRQOL scores [3,18].

Two methods are commonly used to determine MID: distribution-based and anchor-based approaches $[3,19]$. Distribution-based approaches rely on statistical properties of the sample (e.g., variation of score distribution) or the instrument (e.g., measurement precision of scale) to establish clinically meaningful change [19]. Anchor-based approaches assess the extent to which changes in measurement instruments correspond to a minimally important change defined by external indicators. These indicators may include clinical variables (e.g., laboratory and physiological measures and clinical ratings) and patientreported outcomes (PRO) (e.g., global change in health) [20].

To date, there is no consensus on the best approach to evaluate MID $[3,19]$. Studies have recommended that MID estimations should apply anchored-based approaches using clinical and/or PRO indicators combined with supportive information from the distributionbased estimates to generate a small range of values for MID [20-23]. The strength of using multiple approaches to establish a range of MID is to demonstrate variability among estimates.

The main purpose of this study was to validate and interpret a Taiwanese version of the DQOL [24]. We evaluated the psychometric properties of the DQOL using several clinical variables: 1) laboratory indicators: fasting plasma glucose, 2-hour postprandial plasma glucose, and HbA1c, and 2) complications of diabetes: retinopathy, neuropa- 
thy, diabetic foot disorder, cardiovascular, and cerebrovascular diseases. To better interpret the DQOL, we estimated the MID using a combined anchor-based and distribution-based approach.

\section{Methods \\ Participants and data collection}

Data were collected from the Taiwan Diabetes Health Survey, an initiative to systematically develop HRQOL instruments for diabetes patients. In the second-year of the project, we focused on the DQOL and assessed its psychometric properties. Face-to-face interviews were conducted by two trained research associates for type- 1 and type- 2 diabetes patients who utilized outpatient services in the Tainan Hospital - a Taiwan's Department of Health (DOH) affiliated teaching hospital - between 07/200610/2006. In total, data from 337 diabetes patients were collected for the statistical analysis. This study was approved by the Institutional Review Board of the Tainan Hospital and received informed consent from each patient.

Data on laboratory measure, clinical diagnosis and HRQOL assessment were collected at the same time from individual patients and tested using the same methods for all patients. Laboratory indicators include fasting plasma glucose (FPG), 2-hour postprandial plasma glucose (2 h PPG), and HbA1c. Diabetes complications were abstracted from medical records, including retinopathy (none vs. background, proliferative, or decreased vision), neuropathy (none vs. present), diabetic foot disorders (none vs. foot ulceration, sepsis, or amputation), cardiovascular complications (none vs. angina, or previous myocardial infarction or congestive heart failure), and cerebrovascular complications (none vs. transient ischemic attack, or stroke).

\section{Background of developing the DQOL}

The DQOL was originally developed to assess HRQOL for type-1 diabetes [11] and has been adapted for type-2 diabetes [25-27]. The original DQOL consists of 46 items measuring the domains of satisfaction with treatment, impact of treatment, worry about future effects of diabetes, and worry about social/vocational issues [11]. The DQOL has been translated to Chinese language for people in Canada [9], with a modification of the original instrument (i.e., adding and replacing some items) to capture culture-sensitive issues such as eating and sexual activities. These modifications are necessary because eating style and joyfulness are essential components of Chinese culture where family gathering and social activities are centered on meals. By contrast, sexual activity is a taboo subject in Chinese culture especially among elderly people who are less willing to reported sexual functioning. Our previous study suggests that measuring sexual functioning by dia- betes elderly people is less reliable and less valid compared to other diabetes HRQOL domains [7].

The extant Chinese version developed for Chinese people in Canada can not be directly applied to our study population because different spoken dialects and syntax (i.e., using different rules and principles to govern the sentence structure) are used by Chinese people in Canada and Taiwan. The extant DQOL in Chinese language was developed based on the dialect of Cantonese [9], where people in Taiwan use Mandarin Chinese. To address this issue, we included all items of the extant Chinese version form Canada [9], but explicitly modified syntax of individual item. For example, we replaced an item "您對於自己染上糖尿病併發症的憂慮有多頻密"

of the extant Chinese version from Canada by the item "您常常擔心糖尿病的併發症嗎" for Taiwanese. After item modification and replacement, we translated our Taiwanese version of the DQOL back to an English version [25] and compared the semantics of the translated English version to the original English version. We also invited seven diabetes patients (four males and three males; age range 60-80 years) from the same hospital and applied cognitive debriefing tests to assess the level of comprehension and cognitive equivalence of the items. The finding from cognitive debriefing tests suggests a minor revision in the wordings for some items.

This Taiwanese version includes the same items as those in a Chinese version developed in Canada [9]. Compared to the original DQOL, for satisfaction domain, we dropped one item asking about sexual life (How satisfied are you with your sex life?), and replaced it with a new item for diabetes control (How satisfied are you with your control over your diabetes?). For impact domain, we dropped two items asking about interference with sexual life (How often does your diabetes interfere with your sex life?) and insulin reactions (How often do you hide from others the fact that you are having an insulin reaction?). We replaced them with two new items on eating out (How often does your diabetes interfere with your eating out?) and traveling/vacation (How often do you avoid a vacation or trip because of your diabetes?). For worry domain, consistent with a Chinese version from Canada we dropped seven items asking about social/vocational worry associated with marriage, children, education, job, and insurance because these items are appropriate for younger adults. We, however, added three items relevant to worry about requiring insulin in the future (How often do you worry about requiring insulin in the future?), death (How often do you worry about death due to diabetes?), and eating food (How often do you worry about eating the 
wrong food?). The resulting version of the DQOL consisted of 42 items measuring three domains: 15 items for the satisfaction with treatment domain, 20 items for the impact of treatment domain, and 7 items for the worry about future effect of diabetes domain. Our factor analysis suggests a goodness-of-fit for the factorial structures of the translated DQOL [24]. The detailed process of developing a Taiwanese version of the DQOL has been described in our previous study [24].

All items are scored on a five-point Likert scale, ranging from 1 (very satisfied) to 5 (very dissatisfied) in satisfaction domain, and from 1 (never) to 5 (all the time) in impact and worry domains. Domain scores were calculated by summing responses of all items in the corresponding domains, and lineally transforming them to a 1-100 scale with higher scores representing poorer HRQOL. A summary score (overall HRQOL) is further derived by summing three domain scores and lineally transforming to a $1-100$ scale.

\section{Other HRQOL measures: the D-39S and the RAND-I 2}

We collected other HRQOL measures to validate the DQOL, including the D-39S and the RAND-12. The D-39S is a short-form (23 items) of the D-39, which is a diabetesspecific HRQOL instrument designed for patients with type- 1 and type-2 diabetes [10]. The D-39 has been translated to Chinese language by our research team and demonstrates good psychometric properties [7]. We shortened the D-39 using the Ant Colony Algorithm and structural equation modeling, which specifically retained items showing best correlation with clinical variables and goodness-of-fit for the construct of interest [28]. The D-39S covers the same domains as the D-39: energy and mobility, diabetes control, anxiety and worry, social burden, and sexual functioning. Items are administered using seven response categories with score ranging from 1 (not affected at all) to 7 (extremely affected). Domain scores are calculated by summing all items in the same domain, and linearly transformed them to $1-100$, with high scores representing poor HRQOL.

The RAND-12, a generic HRQOL instrument, is a shortform of the RAND-36 [29]. The RAND-12 uses 12 items to capture two underlying constructs: physical and mental health. We calculated two summary scores, a physical health composite (PHC) and mental health composite (MHC), which are norm-based standardized scores with a mean 50 and a standard deviation 10. Higher scores in PHC and MHC represent better HRQOL. We used the RAND-12 PHC and MHC instead of the SF-12 physical component score (PCS) and mental component score (MCS) because evidence suggests that the SF-12 might be less sensitive to detect important difference in HRQOL between the known groups [30].

\section{Psychometric analyses for the DQOL}

Psychometric properties of the DQOL were examined using internal consistency (reliability), convergent/discriminant validity, and known-groups validity.

Internal consistency of each domain was estimated using Cronbach's alpha coefficient. An alpha of $\geq 0.7$ is considered to be acceptable for the purpose of group comparisons [31]. Convergent and discriminant validity was assessed through a multi-trait multi-method (MTMM) which compares Pearson's correlation coefficients among domains of the DQOL with the D-39S and the RAND-12. As described in the Introduction, because the DQOL essentially measures satisfaction and impact of diabetes treatment, whereas the D-39S measures physical functioning and psychological well-being associated with diabetes, we hypothesized that the two DQOL domains (satisfaction with treatment and impact of treatment) would be more strongly associated with physical domains of the D395 (diabetes control and energy and mobility) compared to with psychosocial domains of the D-39S (social burden, anxiety and worry, and sexual functioning). We also hypothesized that the worry domain of the DQOL which focuses more on physical aspects (such as worry about complication, change of physical appearance and death) would be strongly associated with physical domains of the D-39S (diabetes control and energy and mobility) compared to with psychosocial domains of the D-39S (social burden, anxiety and worry, and sexual functioning). With respect to the association between the DQOL and the RAND-12, we assumed that the DQOL domains would be more strongly associated with $\mathrm{PHC}$ compared to with MHC. A magnitude of Pearson's correlation coefficient $0-0.39,0.4-0.69$, and $\geq 0.7$ is classified as weak, moderate, and strong, respectively [31].

Known-groups validity of the DQOL was examined by the extent to which the DQOL can discriminate between clinically well-defined patient groups, including laboratory diagnosis and diabetic complication groups. Laboratory diagnosis known groups are for those patients whose values of laboratory measures were below vs. above the accepted cut-off points: $110 \mathrm{mg} / \mathrm{dL}$ for FPG, $140 \mathrm{mg} / \mathrm{dL}$ for $2 \mathrm{~h} \mathrm{PPG}$, and 7.0\% for HbA1c [32]. Diabetes complication known groups are for patients who were diagnosed with vs. without complications of retinopathy, neuropathy, diabetic foot diseases, cardiovascular, and cerebrovascular diseases, respectively. We calculated Cohen's effect size (ES) to indicate the magnitude of known-groups validity (unit: standard deviation [SD]) [31], defined as the differences in domain scores between known groups (e.g., HbA1c below vs. above 7.0\%) divided by the pooled standard deviation of both groups. A magnitude of effect size $<0.2$ SD, 0.2-0.49 SD, 0.5-0.79 SD, and $\geq 0.8$ SD is classified as negligible, small, moderate, and large, respec- 
tively. We hypothesized that, compared to the RAND-12 the DQOL and the D-39S would discriminate better between laboratory known groups (with a ES $\geq 0.2$ ) because these two instruments center on burden of diabetes treatment and symptoms of glycemic control. We also hypothesized that, compared to the RAND-12 the DQOL and the D-39S would discriminate better between the known groups of retinopathy, neuropathy, and diabetic foot complications rather than the known groups of cardiovascular and cerebrovascular complications. This is because these three complications are closely associated with diabetes treatment and control, and their impact could be directly captured by the domains included in both diabetes-specific instruments.

We also compared scores of the DQOL domains by treatment regimens, including 1) lifestyle modification alone or lifestyle modification plus oral agent (L/LO) and 2) lifestyle modification plus insulin or lifestyle modification plus oral agent and insulin (LI/LOI). We hypothesized that patients who were treated with $\mathrm{L} / \mathrm{LO}$ regimen would demonstrate better HRQOL compared to patients who were treated with LI/LOI regimen.

\section{Establishment of minimally important difference}

We used a cross-sectional method to determine MID which compares HRQOL scores in patients who were classified by level of health-relevant criteria $[3,19]$. Because there is no consensus on the superiority of a anchor- vs. distribution-based approach to determine MID (also see Introduction), we specifically combined the findings using a anchor-based approach (differences between health distinguishable groups) with a distribution-based approach [21,23].

Three-single items measuring patient's self-reported diabetes severity, general health status, and global quality of life were considered as anchors. Items of diabetes severity and global quality of life were rated by a seven categories, with scores ranging from 1 to 7 (from most severe/very dissatisfied to least severe/very satisfied). The item of general health status was rated by a five categories, with score ranging from 1 to 5 (poor, fair, good, very good, and excellent). We estimated differences in average HRQOL scores across adjacent categories of a specific anchor $[21,23,33]$. We considered the MID to be the difference in average scores corresponding to the effect size between 0.2 and $0.5[23,34]$.

For a distribution-based approach, we estimated a standard error of measurement (SEM) which accounts for reliability of the DQOL and standard deviation of patients under the investigation. SEM was estimated by a standard deviation of the DQOL scores multiplied by a square root of one minus internal consistency of the DQOL scores.
Based on evidence supported by Wyrwich and colleagues, we adopted a one-SEM criterion to reflect MID $[35,36]$. We finally used the findings derived from three anchors and a SEM to generate a range of MID values for individual DQOL domain.

In this study, all of the analyses were performed using the STATA 9.02 [37].

\section{Results}

\section{Patient characteristics}

Table 1 shows patients' characteristics $(\mathrm{N}=337)$. Briefly, mean age was 61.6 years (SD: 10.9 ) and $51 \%$ were male. For laboratory indicators, mean FPG was $151 \mathrm{mg} / \mathrm{dL}$ (SD: 48), mean 2 h PPG was $204 \mathrm{mg} / \mathrm{dL}$ (SD: 78), and mean HbA1c was $7.9 \%$ (SD: 2.0). For diabetes complications, $16 \%$ had retinopathy, $14 \%$ had cardiovascular disease, $13 \%$ had diabetic foot disorder, $13 \%$ had neuropathy, and $5 \%$ had cerebrovascular disease. The majority of the subjects $(88 \%)$ were treated with lifestyle modification or lifestyle modification plus oral agent.

\section{Internal consistency}

Internal consistency was $\geq 0.7$ for all domains of the DQOL $(0.90,0.89$, and 0.83 for impact, satisfaction, and worry domains, respectively).

\section{Convergent/discriminant validity}

Table 2 shows convergent and discriminant validity of the DQOL against the D-39S and the RAND-12. In general, domain scores of the DQOL were moderately correlated with the D-39S (except sexual functioning) and the RAND-12 PHC and MHC, with Pearson's correlation coefficients $\geq 0.4$. Magnitudes in the correlations of all DQOL domains with the diabetes control and energy/mobility of the D-39S were slightly larger than with the other D-39S domains (social burden, anxiety and worry, and sexual functioning). For example, Pearson's correlation coefficients of the satisfaction domain of the DQOL with diabetes control and energy/mobility of the D-39S were all 0.57 , which were larger than with the other D-39 domains (0.30 through 0.46$)$. Magnitudes in the correlations of all DQOL domains with the RAND-12 PHC were slightly larger than with the RAND-12 MHC.

\section{Known-groups validity}

Table 3 shows the known-groups validity tested using laboratory indicators and diabetes complications. After adjusting for age, gender, education background, and diabetes duration, the impact, worry, and overall HRQOL domains of the DQOL demonstrated discernible discriminative ability for $2 \mathrm{~h}$ PPG groups (effect sizes in score difference $\geq 0.2$ ), but the satisfaction domain did not (effect size $<0.2$ ). For HbA1c groups, the satisfaction, worry, and overall HRQOL domains of the DQOL demonstrated dis- 
Table I: Patients characteristics $(\mathbf{N}=337)$

\begin{tabular}{|c|c|}
\hline & Mean (SD) or \% \\
\hline \multicolumn{2}{|l|}{ Demographic variables } \\
\hline Age in years, mean (SD) & $61.6(10.9)$ \\
\hline$<55, \%$ & 25.2 \\
\hline $55-59.9, \%$ & 14.0 \\
\hline $60-64.9$ & 19.3 \\
\hline $65-69.9$ & 18.4 \\
\hline $70-74.9$ & 10.4 \\
\hline$\geq 75$ & 12.8 \\
\hline \multicolumn{2}{|l|}{ Gender, \% } \\
\hline Male & 50.7 \\
\hline Female & 49.3 \\
\hline \multicolumn{2}{|l|}{ Education, \% } \\
\hline No formal education & 7.1 \\
\hline Primary and junior high schools & 52.5 \\
\hline Senior high school & 21.4 \\
\hline College & 17.8 \\
\hline Graduate & 1.2 \\
\hline \multicolumn{2}{|l|}{ Employment status, \% } \\
\hline Yes & 40.4 \\
\hline No & 59.6 \\
\hline \multicolumn{2}{|l|}{ Laboratory tests } \\
\hline Fasting plasma glucose (FPG), mg/dL & $150.7(47.8)$ \\
\hline 2-hour postprandial plasma glucose (2 h PPG), mg/dL & $204.0(78.0)$ \\
\hline Hemoglobin Alc (HbAlc), \% & $7.9(2.0)$ \\
\hline \multicolumn{2}{|l|}{ Diabetes complications } \\
\hline Retinopathy, \% & 16.1 \\
\hline Neuropathy, \% & 12.5 \\
\hline Diabetic foot complications, $\%$ & 12.5 \\
\hline Cardiovascular complications, \% & 14.0 \\
\hline Cerebrovascular complications, \% & 5.3 \\
\hline Number of comorbid conditions, mean (SD) & $1.8(1.2)$ \\
\hline Duration of DM in years, mean (SD) & $9.2(6.3)$ \\
\hline \multicolumn{2}{|l|}{ Type of treatment, \% } \\
\hline Lifestyle modification alone or lifestyle modification plus oral agent & 87.9 \\
\hline Lifestyle modification plus insulin or lifestyle modification plus oral agent and insulin & 12.1 \\
\hline \multicolumn{2}{|l|}{ Type of diabetest, \% } \\
\hline Type-I (age<30 years old and BMI<23) & 1.2 \\
\hline Type- 2 (either age $\geq 30$ years old or BMI $\geq 23$, or both) & 98.8 \\
\hline
\end{tabular}

† Classification of type of diabetes $[49,50]$

cernable discriminative ability, but the impact domain did not. Discriminative ability of the DQOL and the D$39 \mathrm{~S}$ by $2 \mathrm{~h}$ PPG and HbA1c known groups was compromised, depending on specific domains. Compared to the RAND-12, both diabetes-specific HRQOL instruments showed slightly better discrimination by using laboratory indicators. No specific domains of the DQOL, the D-39S, and the RAND-12 showed discernible discriminative ability for FPG groups.

For the diabetes complication known groups, after adjusting for age, gender, education background, and diabetes duration, the impact, worry, and overall HRQOL domains of the DQOL demonstrated better discrimination than the satisfaction domain. This is especially evident for the known groups of retinopathy, neuropathy, and diabetic foot complications. Taking neuropathy as an example, the effect sizes in score differences of the impact, worry, and overall HRQOL domains were 0.44, 0.46, and 0.45, respectively, which were larger than the satisfaction domain (0.24). The discriminative ability of the DQOL and the D-39S by the known groups of retinopathy, neuropathy, and diabetic foot complications was compromised. Compared to the RAND-12, both diabetes-specific HRQOL instruments showed slightly better discrimination by laboratory indicators. By contrast, compared to 
Table 2: Convergent/discriminant validity the DQOL $†$

\begin{tabular}{lcccc}
\hline & $\begin{array}{c}\text { DQOL } \\
\text { SAT } \ddagger\end{array}$ & $\begin{array}{c}\text { DQOL } \\
\text { IMP } \ddagger\end{array}$ & $\begin{array}{c}\text { DQOL } \\
\text { WOR } \ddagger\end{array}$ & $\begin{array}{c}\text { DQOL } \\
\text { ALL }\end{array}$ \\
\hline D-39 & & & & \\
Diabetes control & $-0.57 \S$ & -0.66 & -0.54 & -0.70 \\
Energy and mobility & -0.57 & -0.68 & -0.54 & -0.71 \\
Social burden & -0.46 & -0.65 & -0.47 & -0.64 \\
Anxiety and worry & -0.51 & -0.59 & -0.46 & -0.62 \\
Sexual functioning & -0.30 & -0.37 & -0.29 & -0.38 \\
RAND-12 & & & & \\
PHC\# & -0.53 & -0.72 & -0.57 & -0.73 \\
MHC\# & -0.50 & -0.68 & -0.53 & -0.69 \\
\hline
\end{tabular}

† Values in the cells are Pearson's correlation coefficients: weak (0$0.39)$, moderate $(0.40-0.69)$, and strong $(\geq 0.7)$

‡ SAT: satisfaction; IMP: impact; WOR: worry; ALL: overall

$\S$ Negative value in Pearson's correlation coefficients is due to better HRQOL indicated by lower scores for the DQOL and the D-39S and higher scores for the RAND-12

\# PHC: physical health composite; MHC: mental health composite

the RAND-12 the discriminative ability of the DQOL and the D-39S was compromised by cardiovascular complication known groups, but less satisfied by cerebrovascular complication known groups.

\section{Treatment effect}

Figure 1 shows associations of HRQOL and types of diabetes treatments - 1) lifestyle modification alone or lifestyle modification plus oral agent (L/LO) and 2) lifestyle modification plus insulin or lifestyle modification plus oral agent and insulin (LI/LOI). As we hypothesized, after adjusting for age, gender, education, and diabetes duration patients treated by LI/LOI regimen were associated with more impaired HRQOL in all domains than patients treated by L/LO regimen. The effect sizes in the score differences between two regimens for satisfaction, impact, worry, and overall HRQOL domains were all above 0.2 (i.e., $0.20,0.48,0.29$, and 0.39 , respectively), indicating clinically important difference.

\section{Minimally important differences}

Table 4 shows MID for individual DQOL domain estimated using the anchor- and distribution-based approaches. Because case numbers were small for categories 1-3 in the self-reported diabetes severity and global quality of life anchors, these three categories were collapsed. For the self-reported diabetes severity anchor, MIDs (estimated by averaging differences in values between adjacent categories with corresponding effect size $0.2-0.5$ ) were $2.6,4.1,6.5$, and 3.1 points for the domains of satisfaction, impact, worry, and overall HRQOL, respectively. For the general health status anchor, the estimated MIDs were 4.9, 3.6, 5.6, and 3.7 points for the domains of satisfaction, impact, worry, and overall HRQOL, respectively. For the global quality of life anchor, the estimated MIDs were 3.0, 4.5, 7.0, and 4.1 points for the domains of satisfaction, impact, worry, and overall HRQOL, respectively. SEM of the distribution-based approach shows the MIDs were 3.7, 4.6, 8.0, and 3.0 points for the domains of satisfaction, impact, worry, and overall HRQOL, respectively.

Combining the findings from anchor- and distributionbased approaches, the range of MIDs were 3-5 points for the satisfaction domain, 4-5 points for the impact domain, 6-8 points for the worry domain, and 3-4 points for the overall HRQOL.

\section{Discussion}

Although the DQOL has been widely used in many studies [25-27], rigorous psychometric assessments for a Chinese language version are still limited. In this study, we

Table 3: Known-groups validity the DQOL†

\begin{tabular}{|c|c|c|c|c|c|c|c|c|c|c|c|}
\hline & $\begin{array}{l}\text { DQOL } \\
\text { SAT }\end{array}$ & $\begin{array}{l}\text { DQOL } \\
\text { IMP } \ddagger\end{array}$ & $\begin{array}{l}\text { DQOL } \\
\text { WOR } \ddagger\end{array}$ & $\begin{array}{c}\text { DQOL } \\
\text { ALL }\end{array}$ & $\begin{array}{l}\text { D39 } \\
\text { DC } \neq\end{array}$ & $\begin{array}{l}\text { D39 } \\
\text { EM } \ddagger\end{array}$ & $\begin{array}{l}\text { D39 } \\
\text { SB } \neq\end{array}$ & $\begin{array}{l}\text { D39 } \\
\text { AW } \ddagger\end{array}$ & $\begin{array}{l}\text { D39 } \\
\text { SF } \neq\end{array}$ & $\begin{array}{c}\text { RAND- } 12 \\
\text { PHC } \neq\end{array}$ & $\begin{array}{c}\text { RAND-12 } \\
\text { MHC } \neq\end{array}$ \\
\hline \multicolumn{12}{|l|}{ Laboratory indicators } \\
\hline Fasting plasma glucose & $0.04 \S$ & 0.06 & 0.12 & 0.08 & 0.08 & 0.07 & 0.05 & 0 & -0.19 & 0.01 & 0.01 \\
\hline 2-hour postprandial plasma glucose & 0.17 & 0.24 & 0.34 & 0.28 & 0.39 & 0.40 & 0.26 & 0.21 & 0.43 & 0.34 & 0.20 \\
\hline Hemoglobin Alc & 0.23 & 0.17 & 0.22 & 0.23 & 0.19 & 0.20 & 0.20 & 0.14 & -0.04 & 0.15 & 0.11 \\
\hline \multicolumn{12}{|l|}{ Diabetes complications } \\
\hline Retinopathy & 0.10 & 0.42 & 0.31 & 0.35 & 0.36 & 0.48 & 0.41 & 0.47 & 0.31 & 0.30 & 0.07 \\
\hline Neuropathy & 0.24 & 0.44 & 0.46 & 0.45 & 0.49 & 0.57 & 0.64 & 0.43 & 0.44 & 0.49 & 0.24 \\
\hline Diabetic foot complications & 0.08 & 0.39 & 0.34 & 0.33 & 0.45 & 0.60 & 0.59 & 0.47 & 0.40 & 0.50 & 0.23 \\
\hline Cardiovascular complications & -0.06 & 0.20 & 0.14 & 0.13 & 0.21 & 0.32 & 0.15 & 0.18 & 0.32 & 0.27 & 0.13 \\
\hline Cerebrovascular complications & -0.09 & 0.08 & 0.04 & 0.03 & 0.12 & 0.30 & 0.41 & 0.31 & -0.09 & 0.74 & 0.60 \\
\hline
\end{tabular}

† Covariate adjustment: age, gender, education background, and duration

‡ SAT: satisfaction; IMP: impact; WOR: worry; ALL: overall; DC: diabetes control; EM: energy and mobility; SB: social burden; AW: anxiety and worry; SF: sexual functioning; PHC: physical health composite; MHC: mental health composite

$\S$ Values in the cells are effect size: negligible $(<0.2)$, small $(0.2-0.49)$, moderate $(0.5-0.79)$, and large $(\geq 0.8)$ 


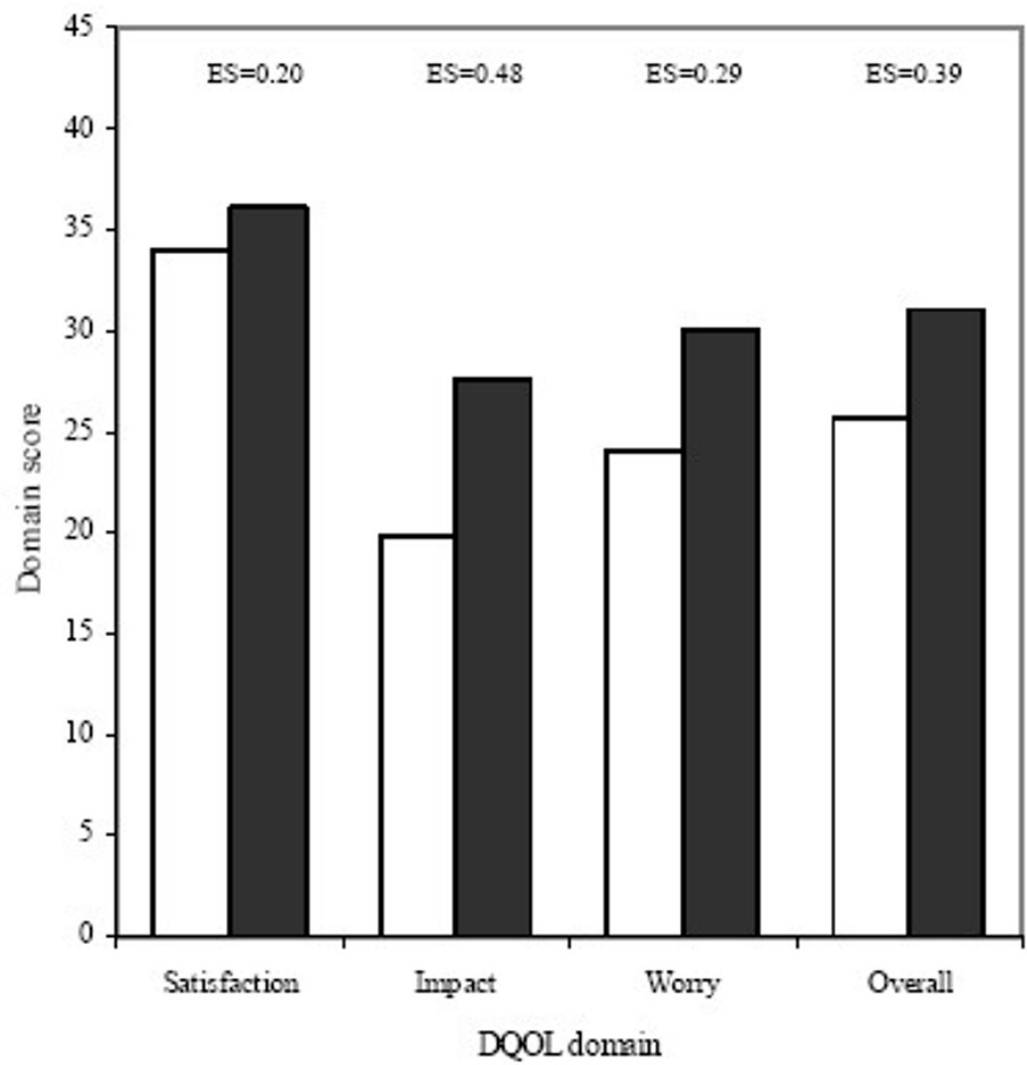

口Lifestyle modification alone or lifestyle modification phus oral agent

口Lifestyle modification phs insulin or lifestyle modification plus oral agent and insulin

Figure I

Relationship between DQOL score and type of treatment. ES: effect size, which is the difference in HRQOL scores (unit: SD) of the DQOL between two treatment categories.

tested psychometric properties of the DQOL in Chinese language for diabetes patients treated in Taiwan. Our version was harmonized with a previous version developed for Chinese people in Canada [9], which may facilitate the use in Chinese populations worldwide.

Our findings indicate that scores of the DQOL were moderately correlated with the D-39S (except sexual functioning) and the RAND-12. Magnitudes in the correlations of all DQOL domains with the physical relevant domains of the D-39S (diabetes control and energy/mobility) were slightly larger than with other domains of the D-39S (social burden, anxiety and worry, and sexual functioning). Additionally, magnitudes in the correlations of all DQOL domains with the RAND-12 PHC were slightly larger than with MHC. A DQOL validation study by Yildirim and colleagues reported that domains scores of the DQOL were more strongly correlated with the physical domains (e.g., mobility, vision, hearing, breathing, and so on) of the 15D (a generic HRQOL measure) than with the psychological domains (e.g., mental function, depression, distress, and so on) [38]. Another DQOL validation study by Jacobson and colleagues also reported that domains scores of the DQOL were more strongly correlated with the physical domains (e.g., role physical functioning and general health) of the SF-36 than with the psychosocial domains (e.g., social functioning) [25]. Taken together, these findings might suggest that the concepts captured by the DQOL, such as satisfaction with treatment, impact of treatment, and worry about future diabetes effects (complications, change of physical appearance and death), are more physical than psychosocial relevant. Therefore, the HRQOL constructs included in the DQOL and the D-39S are not completely equivalent.

For known-groups validity, we found that, compared to the RAND-12 both diabetes-specific HRQOL instruments demonstrated slightly better discrimination by known groups of $2 \mathrm{~h}$ PPG and HbA1c. Additionally, compared to 
Table 4: Minimally important differences of the DQOL

\begin{tabular}{|c|c|c|c|c|c|c|c|c|c|c|c|c|c|}
\hline \multirow[b]{2}{*}{ DQOL } & \multicolumn{4}{|c|}{$\begin{array}{c}\text { Anchor I: } \\
\text { Self-reported diabetes severity }\end{array}$} & \multicolumn{4}{|c|}{$\begin{array}{c}\text { Anchor 2: } \\
\text { General health status }\end{array}$} & \multicolumn{4}{|c|}{$\begin{array}{c}\text { Anchor 3: } \\
\text { global quality of life }\end{array}$} & \multirow[t]{2}{*}{ SEM } \\
\hline & Category & Mean & $\begin{array}{l}\text { Difference } \\
\text { in meant }\end{array}$ & MID§ & Category & Mean & $\begin{array}{l}\text { Difference } \\
\text { in mean }\end{array}$ & MID & Category & Mean & $\begin{array}{l}\text { Difference } \\
\text { in mean }\end{array}$ & MID & \\
\hline \multirow[t]{5}{*}{ SAT\# } & $1-3$ & 39.42 & - & 2.62 & I & 44.33 & - & 4.90 & $1-3$ & 42.21 & - & 2.99 & 3.71 \\
\hline & 4 & 36.33 & $3.12 \ddagger$ & & 2 & 36.58 & 13.24 & & 4 & 36.36 & 5.87 & & \\
\hline & 5 & 33.66 & $2.32 \ddagger$ & & 3 & 32.00 & $5.11 \neq$ & & 5 & 33.23 & $2.99 \ddagger$ & & \\
\hline & 6 & 31.28 & $2.41 \ddagger$ & & 4 & 26.26 & $4.68 \ddagger$ & & 6 & 32.05 & 1.38 & & \\
\hline & 7 & 24.52 & 7.54 & & 5 & 14.38 & 8.48 & & 7 & 23.24 & 8.50 & & \\
\hline \multirow[t]{5}{*}{ IMP\# } & $1-3$ & 32.17 & - & 4.06 & I & 35.58 & - & 3.60 & $1-3$ & 28.82 & - & 4.52 & 4.58 \\
\hline & 4 & 22.17 & 10.26 & & 2 & 22.17 & $3.59 \ddagger$ & & 4 & 22.58 & $5.70 \ddagger$ & & \\
\hline & 5 & 19.42 & $2.88 \ddagger$ & & 3 & 19.05 & 7.94 & & 5 & 20.30 & 2.51 & & \\
\hline & 6 & 15.36 & $4.01 \ddagger$ & & 4 & 10.61 & $3.64 \ddagger$ & & 6 & 15.99 & $4.95 \ddagger$ & & \\
\hline & 7 & 10.28 & $5.29 \ddagger$ & & 5 & 7.81 & 13.74 & & 7 & 12.33 & $2.91 \ddagger$ & & \\
\hline \multirow[t]{5}{*}{ WOR\# } & $1-3$ & 33.93 & - & 6.53 & I & 32.50 & - & 5.62 & $1-3$ & 26.74 & - & 6.97 & 7.97 \\
\hline & 4 & 26.90 & $7.28 \ddagger$ & & 2 & 27.34 & $4.58 \ddagger$ & & 4 & 29.41 & -3.26 & & \\
\hline & 5 & 25.46 & 1.46 & & 3 & 25.98 & 14.02 & & 5 & 23.19 & $6.66 \ddagger$ & & \\
\hline & 6 & 19.78 & $5.79 \ddagger$ & & 4 & 10.63 & 1.89 & & 6 & 21.93 & 2.30 & & \\
\hline & 7 & 10.02 & 10.93 & & 5 & 9.38 & $6.67 \ddagger$ & & 7 & 12.84 & $7.27 \ddagger$ & & \\
\hline \multirow[t]{5}{*}{ ALL\# } & $1-3$ & 35.05 & - & 3.09 & I & 38.19 & - & 3.72 & $1-3$ & 33.25 & - & 4.13 & 3.02 \\
\hline & 4 & 28.02 & 7.22 & & 2 & 28.18 & 7.21 & & 4 & 28.64 & $4.27 \ddagger$ & & \\
\hline & 5 & $25.5 I$ & $2.44 \ddagger$ & & 3 & 24.83 & 7.94 & & 5 & 25.40 & $3.37 \ddagger$ & & \\
\hline & 6 & 21.78 & $3.73 \ddagger$ & & 4 & 16.20 & $3.72 \ddagger$ & & 6 & 22.72 & $3.23 \ddagger$ & & \\
\hline & 7 & 15.32 & 7.03 & & 5 & 10.42 & 10.68 & & 7 & 16.31 & $5.63 \ddagger$ & & \\
\hline
\end{tabular}

† Covariate adjustment: age, gender, education background, and duration

‡ Differences in mean HRQOL scores across adjacent categories with corresponding effect size $0.2-0.5$

$\S$ MID: minimally important difference, which is the average of the differences in mean HRQOL scores across adjacent categories with effect size

$0.2-0.5$

\# SAT: satisfaction; IMP: impact; WOR: worry; ALL: overall

the RAND-12 both diabetes HRQOL instruments (DQOL and D-39S) discriminated better between the known groups of retinopathy, neuropathy, and diabetic foot complications than the known groups of cardiovascular and cerebrovascular complications. This finding may be in part due to the fact that the indicators of $2 \mathrm{~h} P P G$, HbA1c, retinopathy, neuropathy, and diabetic foot complications are closely associated with diabetes treatment and diabetes control, and their impact might be directly captured by the domains included in both diabetes-specific instruments. In contrast to retinopathy, neuropathy, and diabetic foot complications, the impact of cardiovascular and cerebrovascular complications (especially for stroke as an example) on daily functioning is more significant and might not be directly attributed to glycemic control, which could be better captured by the RAND-12. This finding suggests that it might be an ideal approach to use diabetes-specific HRQOL instruments combined with generic HRQOL instruments to fully measure HRQOL burden for diabetes patients $[7,39,40]$.
Our study extends conventional methods used to validate diabetes HRQOL instruments. Previous studies have often used HbA1c as a glycemic control indicator to validate HRQOL instruments. However, evidence is mixed regarding the association between HbA1c and HRQOL $[1,41$ 43 ]. Our results suggest that 2 h PPG may be a more sensitive laboratory indicator to validate HRQOL in patients with diabetes. Rather than averaging blood glucose levels from the preceding 2-3 months, 2 h PPG captures short term fluctuations in metabolic control. Epidemiologic studies have reported that patients with normal HbA1c, but abnormal $2 \mathrm{~h}$ PPG, are more prone to postprandial hyperglycemia, leading to substantially an increased risk of death from macrovascular diseases $[13,44]$. In most cases, PPG levels increase before and faster than FPG [45]. The usefulness of $2 \mathrm{~h}$ PPG over HbA1c and FPG has been demonstrated in our previous study to test validity of diabetes HRQOL measures using the D-39 [7].

A previous DQOL study suggests that patients with diabetes complications tended to report more impaired DQOL scores compared to their counterparts [9]. In this study, 
we support the value of using several individual complications as known groups to validate diabetes HRQOL instruments. We found that the effect sizes in the impact, worry, and overall HRQOL domains were greater than 0.2 for the known groups of retinopathy, neuropathy, and diabetic foot complications, suggesting clinically meaningful difference. Interestingly, the effect sizes in HRQOL scores between levels of laboratory indicators were generally smaller than for the presence and absence of diabetes complications. This may be due, in part, to the fact that clinical symptoms (e.g., hypoglycemia) and events (e.g., diabetic foot) are more evident to patients than laboratory abnormalities, leading to significant impairment on wellbeing.

Our study suggests that patients who received more intensive treatment (lifestyle modification plus insulin or lifestyle modification plus oral agent and insulin) was associated with the more impaired HRQOL in all domains compared to patients who received less intensive treatment (lifestyle modification alone or lifestyle modification plus oral agent). These comparisons were independent of the influence of age, gender, education, and diabetes duration. Johnson et al reported that, using the SF-12 patients on oral medication plus insulin had significantly lower physical and mental health than patients on oral medication, followed by lifestyle modification alone [30]. Similarly findings were also reported by Saito et al using the SF-36 [46]. However, some longitudinal studies reported that HRQOL was not significantly changed after patients taking insulin therapy $[26,43]$. This discrepancy may be due to the fact that some factors, e.g., increased patient education, family support, and decreased hyperglycemic symptoms, may offset the discomfort and problems related to insulin therapy.

To facilitate interpretation of the DQOL scores, we estimated the minimally important difference (MID). MID has been defined as the smallest difference in a HRQOL measure that is perceived by patients as being clinically meaningful $[3,47]$. Importantly, the choice of anchors might influence the MID estimation. Guyatt et al suggested that a useful anchor for MID should be interpretable and moderately correlated with target instruments $[3,48]$. Revicki et al recommended that anchors derived from patient's perspective should be given the most weight because they reflect the intuitive interpretation for the change in patient-reported outcomes [20]. In this study, three anchors we used (i.e., patient's self-reported diabetes severity, general health status, and global quality of life) were all based on patient's viewpoint. We specifically found that when the levels of external indicators indicated impairment, HRQOL measured by the DQOL showed impairment. Furthermore, these indicators were moderately correlated with the DOQL scores (Pearson's correlation coefficients $>0.4$ ). As a result, we consider these indicators to be legitimate anchors and potentially be useful by other studies to interpret diabetes HRQOL measures.

Because neither anchor-based nor distribution-based approaches are superior to one another, we estimated MIDs based on several anchors and combined these estimates with a distribution-based estimate (i.e., standard error of measurement) [21-23]. We found that the range of MIDs for the DQOL were 3-5 points for satisfaction domain, 4-5 points for impact domain, 6-8 points for worry domain, and 3-4 points for overall HRQOL. The estimation of MIDs can be helpful for calculating sample size when HRQOL is used as an end point in clinical investigations. Interestingly, the findings from this and earlier studies [23] suggest that the combined use of anchor-based and distribution-based approaches tend to expand the range of MIDs compared to using either approach. The MID derived from the distribution-based approach is more likely to be on the opposite end of the MID range compared to the anchor-based approach. More studies using different anchor and distribution-based approaches need to be conducted to confirm these findings.

There are a number of limitations to this study. First, the generalizability of our results may be limited because our samples were collected from a single center in Taiwan. Second, although the DQOL was designed for measuring patients with type- 1 and type- 2 diabetes, only 4 patients in our sample had type-1 diabetes. Therefore, the psychometric properties of DQOL are based largely on type-2 diabetes. Further studies are needed to replicate our finding in patients with type- 1 diabetes. Third, we estimated MIDs using a cross-sectional rather than a longitudinal design. We calculated differences in average HRQOL scores across adjacent categories of an anchor for MID estimations $[21,23,33]$. However, the resulting differences between adjacent groups by a cross-sectional design may not accurately reflect longitudinal changes within the same group. This latter method is known as the minimally important change (MIC) or responsiveness $[3,19]$. A longitudinal design would be preferable approach to examine changes in HRQOL.

\section{Conclusion}

There is a great need to develop and validate diabetes HRQOL instruments for Chinese populations. In this study, we validated a Taiwanese version of the DQOL in Chinese language for diabetes patients in Taiwan. We used different psychometric methods together with different laboratory indicators and diabetes complications to validate the DQOL. In addition to providing a useful questionnaire, we also used a combined anchor-based 
and distribution-based method to interpret the DQOL scores. Further evaluation and improvement are indicated, especially to estimate responsiveness.

\section{Competing interests}

The authors declare that they have no competing interests.

\section{Authors' contributions}

$\mathrm{IH}, \mathrm{JL}$, and $\mathrm{CH}$ conceived the study and its design. MW and $\mathrm{CH}$ collected the data in Taiwan. $\mathrm{IH}, \mathrm{JH}$, and $\mathrm{WL}$ analyzed the data. $\mathrm{IH}, \mathrm{JL}$, and $\mathrm{CH}$ interpreted the data. $\mathrm{IH}$ drafted the manuscript. $\mathrm{IH}, \mathrm{JL}, \mathrm{AW}, \mathrm{MW}, \mathrm{WL}$, and $\mathrm{CH}$ revised critically for important intellectual content.

\section{Acknowledgements}

We thank Dr. Alan M. Jacobson for providing original DQOL and Dr. Alice $Y$. Chang for assistance in the translation of Chinese version of the DQOL. This study was supported by a grant from the Taiwan Department of Health (DOH), under contract \# DOH-9508.

\section{References}

I. Weinberger M, Kirkman MS, Samsa GP, Cowper PA, Shortliffe EA, Simel DL, Feussner JR: The relationship between glycemic control and health-related quality of life in patients with noninsulin-dependent diabetes mellitus. Med Care 1994, 32(I2): I|73-II8I.

2. Patrick DL, Deyo RA: Generic and disease-specific measures in assessing health status and quality of life. Med Care 1989, 27(3 Suppl):S217-32.

3. Guyatt GH, Osoba D, Wu AW, Wyrwich KW, Norman GR, Clinical Significance Consensus Meeting Group: Methods to explain the clinical significance of health status measures. Mayo Clin Proc 2002, 77(4):37I-383.

4. Wild S, Roglic G, Green A, Sicree R, King H: Global prevalence of diabetes: estimates for the year $\mathbf{2 0 0 0}$ and projections for 2030. Diabetes Care 2004, 27(5):1047- 1053.

5. Garratt AM, Schmidt L, Fitzpatrick R: Patient-assessed health outcome measures for diabetes: a structured review. Diabet Med 2002, 19(1): I-II.

6. Watkins K, Connell CM: Measurement of health-related QOL in diabetes mellitus. Pharmacoeconomics 2004, 22(I 7): I I09-। I 26.

7. Huang IC, Hwang CC, Wu MY, Lin W, Leite W, Wu AW: Diabetesspecific or generic measures for health-related quality of life? Evidence from psychometric validation of the D-39 and SF36. Value Health 2008, II(3):450-46I.

8. $\mathrm{Li} \mathrm{TC}$, Lin CC, Liu CS, Li Cl, Lee YD: Validation of the Chinese version of the diabetes impact measurement scales amongst people suffering from diabetes. Qual Life Res 2006, I5(I0): I66-I72.

9. Cheng AY, Tsui EY, Hanley AJ, Zinman B: Developing a quality of life measure for Chinese patients with diabetes. Diabetes Res Clin Pract 1999, 46(3):259-267.

10. Boyer JG, Earp JA: The development of an instrument for assessing the quality of life of people with diabetes. Diabetes39. Med Care 1997, 35(5):440-453.

11. The Diabetes Control and Complications Trial: Reliability and validity of a diabetes quality-of-life measure for the diabetes control and complications trial (DCCT). The DCCT Research Group. Diabetes Care 1988, II (9):725-732.

12. Rubin RR, Peyrot M: Quality of life and diabetes. Diabetes Metab Res Rev 1999, I5(3):205-218.

13. Ceriello A: Postprandial hyperglycemia and diabetes complications: is it time to treat? Diabetes 2005, 54(I): I-7.

14. Monnier L, Mas E, Ginet C, Michel F, Villon L, Cristol JP, Colette C: Activation of oxidative stress by acute glucose fluctuations compared with sustained chronic hyperglycemia in patients with type 2 diabetes. JAMA 2006, 295(14):168I-1687.

15. Lloyd CE, Matthews KA, Wing RR, Orchard TJ: Psychosocial factors and complications of IDDM. The Pittsburgh Epidemiol- ogy of Diabetes Complications Study. VIII. Diabetes Care 1992, I5(2): I66-172.

16. Maddigan SL, Feeny DH, Johnson JA: Health-related quality of life deficits associated with diabetes and comorbidities in a Canadian National Population Health Survey. Qual Life Res 2005, I4(5): | 3||$-\mid 320$.

17. Luscombe FA: Health-related quality of life measurement in type 2 diabetes. Value Health 2000, 3(SuppI I):15-28.

18. Jaeschke R, Singer J, Guyatt GH: Measurement of health status. Ascertaining the minimal clinically important difference. Control Clin Trials 1989, 10(4):407-415.

19. Crosby RD, Kolotkin RL, Williams GR: Defining clinically meaningful change in health-related quality of life. J Clin Epidemiol 2003, 56(5):395-407.

20. Revicki DA, Cella D, Hays RD, Sloan JA, Lenderking WR, Aaronson NK: Responsiveness and minimal important differences for patient reported outcomes. Health Qual Life Outcomes 2006, 4:70.

21. Cella D, Eton DT, Fairclough DL, Bonomi P, Heyes AE, Silberman C, Wolf MK, Johnson DH: What is a clinically meaningful change on the Functional Assessment of Cancer Therapy-Lung (FACT-L) Questionnaire? Results from Eastern Cooperative Oncology Group (ECOG) Study 5592. J Clin Epidemiol 2002, 55(3):285-295.

22. Cella D, Eton DT, Lai JS, Peterman AH, Merkel DE: Combining anchor and distribution-based methods to derive minimal clinically important differences on the Functional Assessment of Cancer Therapy (FACT) anemia and fatigue scales. J Pain Symptom Manage 2002, 24(6):547-56I.

23. Yost KJ, Cella D, Chawla A, Holmgren E, Eton DT, Ayanian JZ, West DW: Minimally important differences were estimated for the Functional Assessment of Cancer Therapy-Colorectal (FACT-C) instrument using a combination of distributionand anchor-based approaches. J Clin Epidemiol 2005, 58(I2): $|24|-|25|$.

24. Liu JH, Huang IC, Hwang CC, Wu AW: Translation and validation of a Chinese version of the Diabetes Quality of Life (DQOL) instrument. International Society for Quality of Life Research meeting abstracts. Qual Life Res 2007, A-73:.

25. Jacobson AM, de Groot M, Samson JA: The evaluation of two measures of quality of life in patients with type I and type II diabetes. Diabetes Care 1994, I 7(4):267-274.

26. Davis TM, Clifford RM, Davis WA, Fremantle Diabetes Study: Effect of insulin therapy on quality of life in Type 2 diabetes mellitus: The Fremantle Diabetes Study. Diabetes Res Clin Pract 200I, 52(I):63-7I.

27. Chesla CA, Fisher L, Mullan JT, Skaff MM, Gardiner P, Chun K, Kanter $\mathrm{R}$ : Family and disease management in African-American patients with type 2 diabetes. Diabetes Care 2004, 27( I 2):2850-2855.

28. Leite WL, Huang IC, Marcoulides GA: Item selection for the development of short-forms of scaling using an Ant Colony Optimization algorithm. Multivar Behav Res 2008, 43(3):4 I I-43 I.

29. Hays RD, Prince-Embury S, Chen H: Rand-36 health status inventory San Antonio, CA: Psychological Corporation; 1998.

30. Johnson JA, Maddigan SL: Performance of the RAND-I 2 and SF12 summary scores in type 2 diabetes. Qual Life Res 2004, I3(2):449-456.

31. Fayers PM, Machin D: Quality of life : the assessment, analysis, and interpretation of patient-reported outcomes Chichester; Hoboken, NJ: J. Wiley; 2007.

32. American Diabetes Association: Standards of medical care in diabetes-2006. Diabetes Care 2006, 29(SuppI I):S4-42.

33. Deyo RA, Inui TS, Leininger J, Overman S: Physical and psychosocial function in rheumatoid arthritis. Clinical use of a selfadministered health status instrument. Arch Intern Med 1982, I 42(5):879-882.

34. Cohen J: Statistical power analysis for the behavioral sciences Hillsdale, NJ: L. Erlbaum Associates; 1988.

35. Wyrwich KW, Nienaber NA, Tierney WM, Wolinsky FD: Linking clinical relevance and statistical significance in evaluating intra-individual changes in health-related quality of life. Med Care 1999, 37(5):469-478.

36. Wyrwich KW, Tierney WM, Wolinsky FD: Further evidence supporting an SEM-based criterion for identifying meaningful 
intra-individual changes in health-related quality of life. J Clin Epidemiol 1999, 52(9):86I-873.

37. STATCorp: Stata Statistical Software: Release 9.0 College Station, TX: Stata Corporation; 2005.

38. Yildirim A, Akinci F, Gozu H, Sargin H, Orbay E, Sargin M: Translation, cultural adaptation, cross-validation of the Turkish diabetes quality-of-life (DQOL) measure. Qual Life Res 2007, 16(5):873-879.

39. Anderson RM, Fitzgerald JT, Wisdom K, Davis WK, Hiss RG: A comparison of global versus disease-specific quality-of-life measures in patients with NIDDM. Diabetes Care 1997, 20(3):299-305.

40. Woodcock AJ, Julious SA, Kinmonth AL, Campbell MJ, Diabetes Care From Diagnosis Group: Problems with the performance of the SF-36 among people with type 2 diabetes in general practice. Qual Life Res 200I, I0(8):66I-670.

41. Testa MA, Simonson DC: Health economic benefits and quality of life during improved glycemic control in patients with type 2 diabetes mellitus: a randomized, controlled, double-blind trial. JAMA 1998, 280( I7): |490-| 496.

42. Wikblad K, Leksell J, Wibell L: Health-related quality of life in relation to metabolic control and late complications in patients with insulin dependent diabetes mellitus. Qual Life Res 1996, 5(I): 123-130.

43. U.K. Prospective Diabetes Study Group: Quality of life in type 2 diabetic patients is affected by complications but not by intensive policies to improve blood glucose or blood pressure control (UKPDS 37). U.K. Prospective Diabetes Study Group. Diabetes Care 1999, 22(7): I I25-1 I36.

44. Coutinho M, Gerstein HC, Wang Y, Yusuf S: The relationship between glucose and incident cardiovascular events. A metaregression analysis of published data from 20 studies of 95,783 individuals followed for $\mathbf{1 2 . 4}$ years. Diabetes Care 1999, 22(2):233-240.

45. Laakso M: Hyperglycemia and cardiovascular disease in type 2 diabetes. Diabetes 1999, 48(5):937-942.

46. Saito I, Inami F, Ikebe T, Moriwaki C, Tsubakimoto A, Yonemasu K, Ozawa $\mathrm{H}$ : Impact of diabetes on health-related quality of life in a population study in Japan. Diabetes Res Clin Pract 2006, 73(I):5I-57.

47. Guyatt G, Walter S, Norman G: Measuring change over time: assessing the usefulness of evaluative instruments. J Chronic Dis 1987, 40(2): 17|- I78.

48. Guyatt GH: Making sense of quality-of-life data. Med Care 2000, 38(9 Suppl II): 175-9.

49. World Health Organization: Obesity: preventing and managing the global epidemic: report of a WHO consultation Geneva: World Health Organization; 2000.

50. Powers AC: Diabetes Mellitus. In Harrison's principles of internal medicine Edited by: Kasper DL, Harrison TR. New York: McGrawHill, Medical Pub Division; 2005.
Publish with Biomed Central and every scientist can read your work free of charge

"BioMed Central will be the most significant development for disseminating the results of biomedical research in our lifetime. "

Sir Paul Nurse, Cancer Research UK

Your research papers will be:

- available free of charge to the entire biomedical community

- peer reviewed and published immediately upon acceptance

- cited in PubMed and archived on PubMed Central

- yours - you keep the copyright

Submit your manuscript here:

http://www.biomedcentral.com/info/publishing_adv.asp
BiolMedcentral 